\title{
Gulf of Mexico Integrated Science - Tampa Bay Study The Impact of Groundwater and Contaminants on Tampa Bay
}

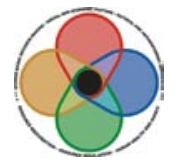

\section{Introduction}

Despite the recreational and economic value of coastal bays and estuaries, these ecosystems are often among our most 'troubled' natural environments. Urbanization, agriculture, mining, and shipping are just a few activities that can have a profound and lasting impact on the coastal zone. In order to maintain a healthy coastal ecosystem, it is crucial to develop reasonable management practices around expert scientific information. We still have much to learn about the quantity and quality of groundwater being discharged into Tampa Bay, Florida. We also need to improve our knowledge of a wide range of contaminants entering the bay and must be able to determine where they accumulate in seafloor sediments. Such buried contaminants can potentially be harmful to biota if they are released to the water column. U. S. Geological Survey (USGS) scientists and research partners from the University of South Florida (USF), the University of Florida (UF), and the Florida Marine Research Institute (FMRI) are mapping sources of groundwater, measuring groundwater flow into Tampa Bay, and assessing the impact of contaminants and sediments on bay water quality and ecosystem health.

\section{Approach}

\section{Groundwater and surface water exchange}

Surface water runoff into the bay can be easily quantified as stream discharge. In the Tampa Bay watershed, however, much of this runoff may contain an additional component called baseflow - or groundwater flow. The abundant and reversible exchange of

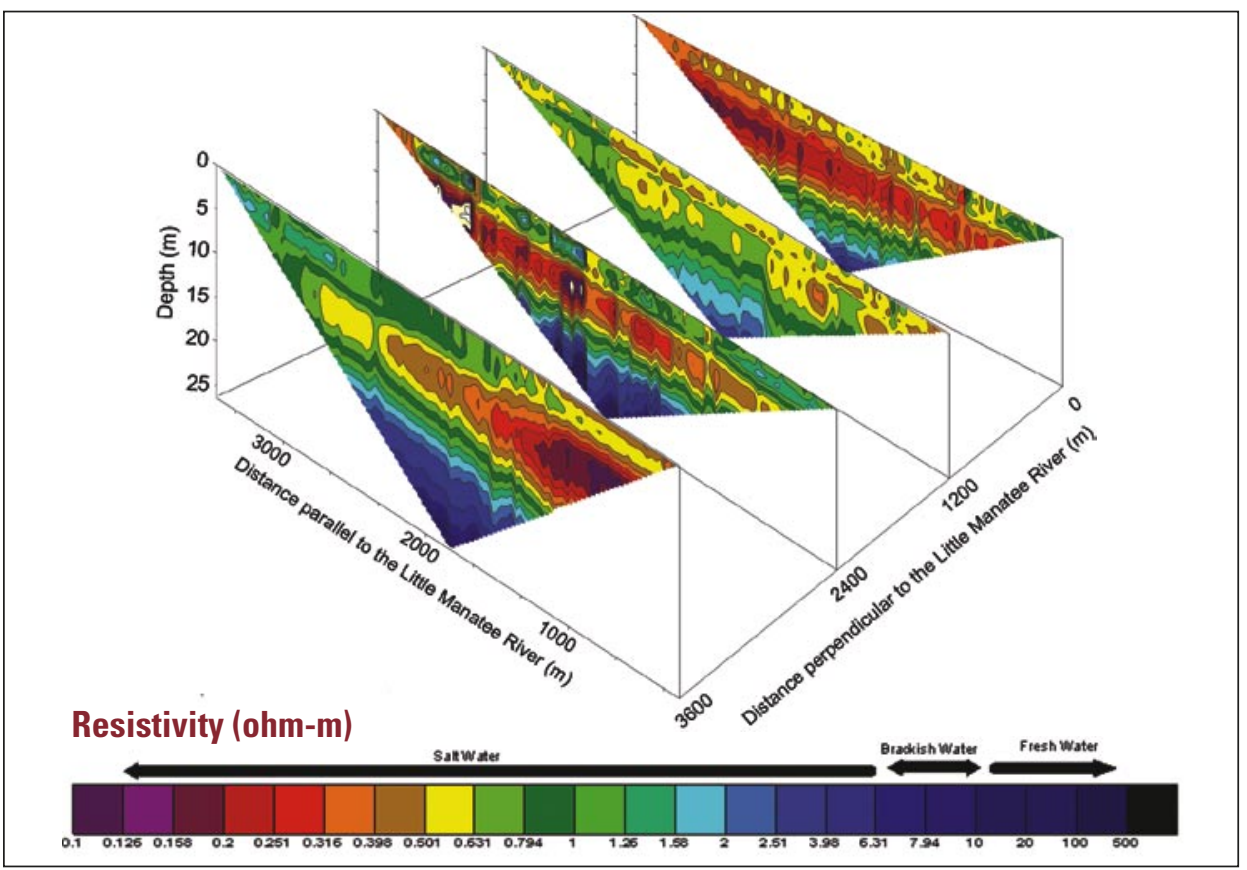

3-D Fence Diagram of Tampa Bay showing saltwater saturation.

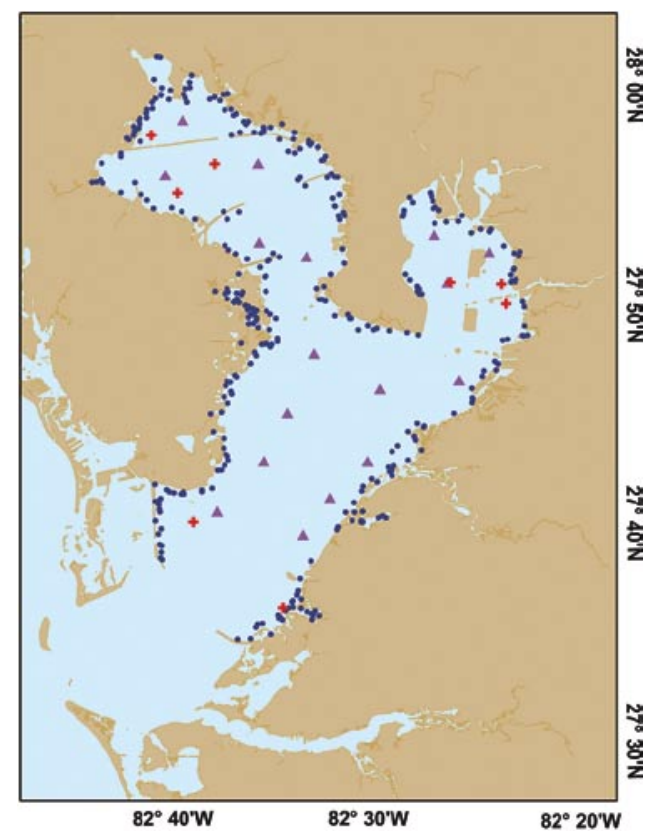

Tampa Bay Study area showing the locations of groundwater and surface water measurements $(\Delta)$, sediment samples (๑), and suspended particulate matter study areas $(+)$.

groundwater and surface water complicates water budget estimates and may also play an important role in the delivery of certain contaminants to the bay. For example, the exchange of water across the sea floor may change the chemical behavior of some contaminants, potentially making them more toxic. To determine the origin of water entering the bay, scientists are conducting a suite of surveys and analyses, such as streaming resistivity mapping. A series of sensors that send an electrical pulse to the seafloor is towed behind a boat. The information conveyed when the electrical pulse bounces back to the sensors can be used to determine if the water below the sea floor is fresh or salty. Measurements of groundwater and 


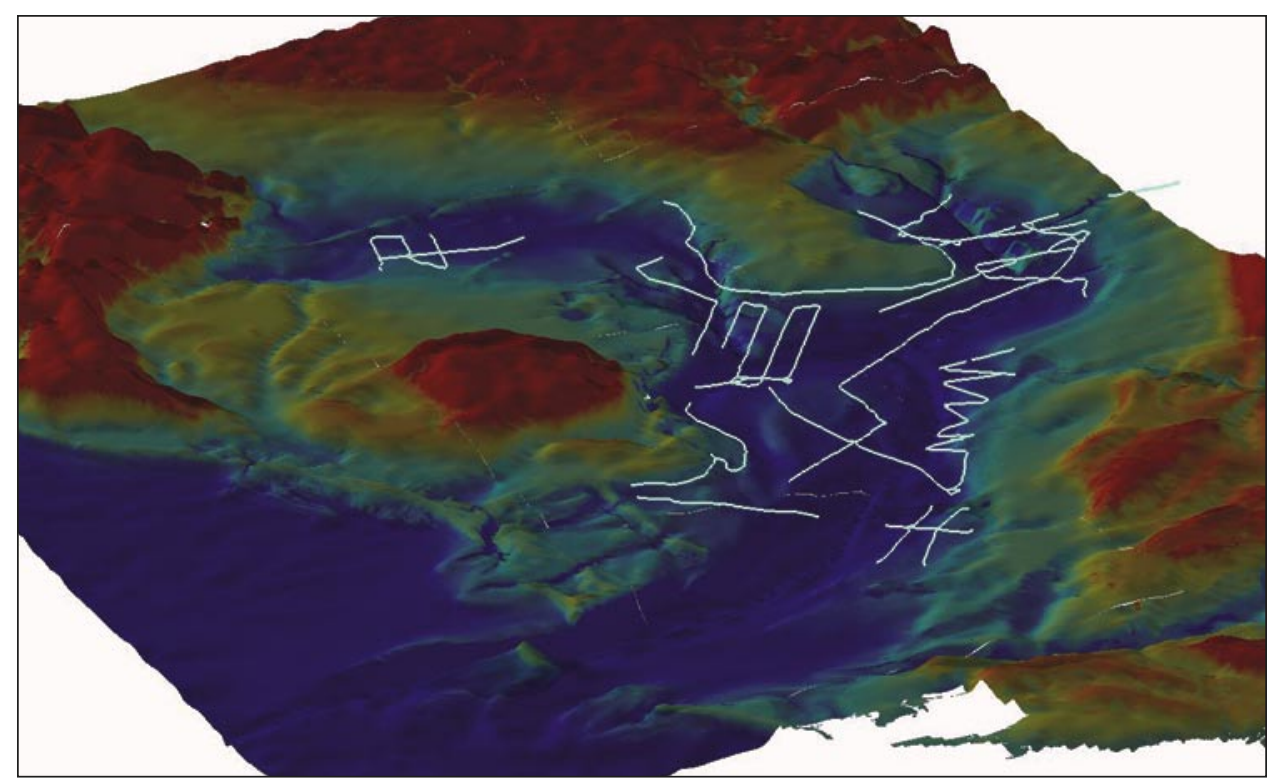

Map depicting the resistivity surveys for 2001 to 2004. Resistivity mapping assists scientists in determining differences in water salinity.

surface water also are being taken to help assess water circulation in Tampa Bay. This information will be used to help develop water circulation, water quality, sediment transport, and habitat distribution models.

\section{Sediments}

USGS scientists are identifying where contaminated sediment is located in Tampa Bay, what types of contaminants (i.e., pesticides, herbicides, and oil and gas byproducts) may be present, and how such contaminants may impact the ecological health of the bay. When bottom sediments are stirred up, they can be carried to other parts of the bay where they may affect the health and distribution of plants and animals. The physical characteristics of sediments, such as their size and type, are also
Role of suspended particulate matter

Suspended particles in the water column of Tampa Bay may consist of resuspended, riverine, or biologically produced particles (i.e., algal blooms). These particles may act as scavengers for reactive contaminants and may also slow down seagrass restoration efforts by attenuating light necessary for photosynthesis. Scientists are identifying the nature of the suspended particulate matter within the bay and are examining the relation between light penetration into the water column and the concentration and composition of suspended particles.

\section{Links to other project research}

Groundwater influx and the availability of contaminants are consistently one of the top management priorities in defining present ecosystem health of Tampa Bay. Research along these themes is closely tied into complementary studies that address 1) the geological evolution of the bay that can provide an historic context from which one can build predictive capabilities and 2) work on the fringing wetlands that can provide insight into the mechanisms of a very effective water/contaminant filter during runoff, and also into the diversity of specialized habitats or ecotones. Multi-faceted maps are being created that can uniquely identify the geologic control on coastal hydrologic processes.
For more information, please contact:

Peter W. Swarzenski, Task Leader,

U.S. Geological Survey, Geologic Discipline

600 Fourth Street South, St. Petersburg, FL 33701

Email: pswarzen@usgs.gov

Kimberly Yates, Project Chief

U.S. Geological Survey, Geologic Discipline

Email:kyates@usgs.gov

\section{Contributing Scientists:}

Paul Carlson, FWC/Florida Marine Research Institute

Darryl L. Felder, University of Louisiana at Lafayette

Mario Fernandez, USGS/Water Resources Discipline

Paul L. Klerks, University of Louisiana at Lafayette

Sarah Kruse, University of South Florida

John Martin, University of Florida

http://gulfsci.usgs.gov 